\title{
Sur la technique des biopsies musculaires (II) Analyse de l'innervation motrice terminale et des plaques motrices en pathologie humaine
}

\author{
Un survol historique
}

\author{
Michel Fardeau, Stéphanie Bauché, Daniel Hantaï
}

L'analyse de l'innervation motrice terminale (IMT) a marqué un premier virage majeur dans l'analyse des biopsies musculaires, jusque là examinées sur coupes en paraffine et colorations histologiques classiques. Ce fut dès 1952, essentiellement grâce aux travaux de Christian Coërs, bientôt suivi par A.L. Woolf [1] utilisant des colorations vitales au Bleu de Méthylène. Ces travaux, entrepris sous l'inspiration de J.G. Greenfield, avaient pour but de mieux différencier les atteintes neuropathiques des atrophies par non-utilisation, ou par cachexie, et des atteintes myopathiques. Ces travaux ont précédé de plusieurs années l'application aux biopsies humaines des techniques cytochimiques et cytoenzymologiques ainsi que les premières études en microscopie électronique.

L'innervation motrice des fibres musculaires avait fait l'objet de travaux classiques de très grande qualité, sur matériel normal ou expérimental, à l'aide des techniques d'imprégnation métallique, en particulier les techniques développées par Bielchowsky. Cela avait permis par exemple à Ranvier de décrire au contact des fibres musculaires une arborisation nerveuse terminale; mais les aspects obtenus avaient fait aussitôt s'opposer les tenants d'une théorie "réticulariste " autour de Boëke, aux tenants de la théorie "neuroniste" soutenue par Ramon y Cajal : les premiers voyaient à l'extrémité nerveuse un "réseau périterminal " anastomosant fibrilles nerveuses et musculaires, alors que, pour les seconds, les rameaux nerveux terminaux ne pénétraient pas sous le sarcolemme des fibres musculaires. La découverte, à l'aide de colorations vitales (au Vert Janus) d'un appareil sous-neural d'aspect lamellaire par René Couteaux fut un argument très fort en faveur de ces derniers. La visualisation d'une activité acétylcholinestérasique au niveau de l'appareil sousneural par Koëlle et Friedenwald (1949), puis les premières images obtenues en microscopie électronique au début des années 1950 sur différents matériels devaient clore ce débat.

Les applications au matériel humain pathologique devenaient alors possibles, en particulier avec les modifications de la technique de Koëlle introduite par Couteaux et Taxi [2]. Demeurait cependant un pré-requis pour l'application de ces techniques au muscle humain : le repérage de la zone d'innervation motrice au cours des prélèvements biopsiques musculaires.

\section{Le repérage de la zone d'innervation motrice}

Dans les muscles squelettiques des mammifères, la zone dans laquelle se terminent les axones moteurs est en règle très étroite, de l'ordre du millimètre, et se situe à mi-distance des extrémités des fibres musculaires, marquées par leur insertion tendineuse ou aponévrotique. La longueur des fibres musculaires étant très variable, et pouvant dépasser plusieurs centimètres dans les muscles humains habituellement biopsiés, un prélèvement " au hasard " n'avait donc que très peu de chances de porter sur la zone d'innervation.

Il fallait donc choisir en premier lieu des muscles dans lesquels les fibres musculaires étaient relativement courtes et leurs extrémités tendineuses faciles à identifier dans le champ opératoire d'un prélèvement à ciel ouvert. Étaient plus particulièrement accessibles, aux membres supérieurs, le chef moyen du muscle deltoïde et le muscle grand palmaire, et, aux membres inférieurs, le muscle court péronier latéral (ont également été étudiés, à des fins 
particulières, le muscle anconé et les muscles intercostaux externes). Pour les autres muscles, et a fortiori pour des muscles souvent biopsiés comme le muscle biceps brachial ou le muscle vaste externe, dont les faisceaux étaient beaucoup plus longs et les extrémités tendineuses non accessibles par une incision cutanée limitée, il convenait donc de recourir à d'autres techniques pour la localisation de cette zone d'innervation.

La première technique utilisée a été électrophysiologique, avec repérage percutané du point moteur, pour les muscles dont les fibres étaient parallèles à la surface cutanée (ce fut en particulier la technique utilisée par C. Coërs). Ce repérage préopératoire peut être complété par une stimulation électrique per-opératoire effectuée dans des conditions compatibles avec l'asepsie chirurgicale; ceci implique une installation particulière de la salle d'opération. Une autre technique, plus simple, et strictement anatomique est possible. En effet, lors de la dissection des faisceaux superficiels d'un muscle à l'aide d'un bistouri à lame pointue et très fine, lorsque l'on passe sur la zone d'innervation la section des filets nerveux moteurs les plus superficiels déclenche un " twitch " très bref, qui donne une localisation très précise de cette zone, au millimètre près; il est recommandé, pour les médecins en charge de ce type de prélèvements biopsiques, d'avoir répété à l'avance cette manipulation sur les muscles d'un petit animal de laboratoire (rat ou souris). Un prélèvement musculaire de 8 à 12 millimètres de long peut alors être réalisé, centré sur la zone repérée.

La dissection des fascicules prélevés se poursuivra sous la loupe binoculaire au laboratoire, avec la confection de blocs "neuraux" contenant les plaques motrices : soit par repérage des filets nerveux terminaux avant ou après fixation par l'acide osmique (M. Fardeau) [3], soit par visualisation des plaques motrices par la technique de Koëlle sur l'un des faisceaux prélevés mis en parallèle des faisceaux sur lesquels seront effectués les prélèvements "neuraux" (A.G. Engel) [4]. La confirmation de la présence des plaques motrices sera apportée par l'examen des coupes semi-fines obtenues à partir de ces blocs "neuraux" (Figure 1).

\section{Visualisation des plaques motrices et de l'innervation motrice terminale}

1. La première méthode utilisée a donc été une coloration vitale par le Bleu de Méthylène. Elle consiste en l'injection du fascicule prélevé, de 2 à 3 millimètres de large, par une solution à 0,03\% de Bleu de

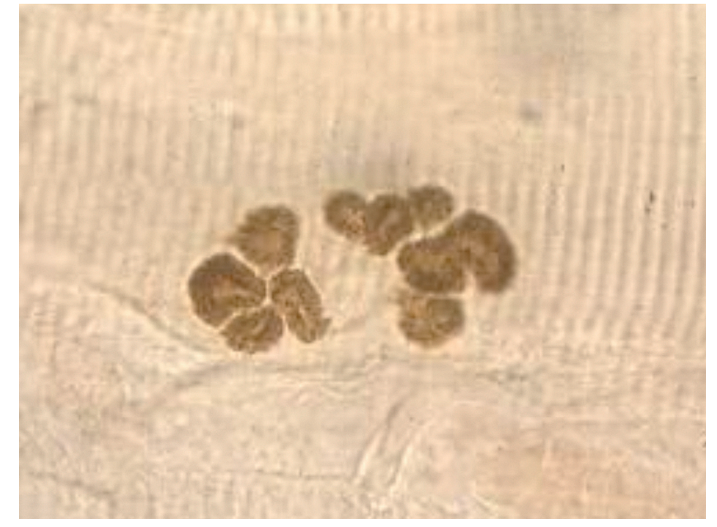

Figure 1

Muscle jumeau interne. Plaque motrice normale. Technique de Koëlle modifiée [2]

Méthylène dissous dans du sérum physiologique, jusqu'à ce que le fascicule devienne "aussi bleu que possible". Il est ensuite placé sous flux d'oxygène, puis fixé dans une solution de molybdate d'ammonium pendant 24 heures à $4{ }^{\circ} \mathrm{C}$. Il est ensuite fixé par le formol à $10 \%$ pendant 24 heures avant d'être débité en série en coupes à congélation de 50 à 100 microns d'épaisseur. Cette technique n'est plus guère utilisée aujourd'hui que dans des conditions expérimentales.

2. La visualisation des plaques motrices s'effectue par la mise en évidence de l'activité acétylcholinestérasique (AChE) des appareils sous-neuraux selon la méthode de Koëlle, adaptée par Couteaux et Taxi [2].

Cette technique peut s'effectuer sur coupes congelées des prélèvements effectués, ou sur dilacérats de quelques fascicules musculaires après une courte fixation formolée (4 à 6 heures). Elle comporte deux temps, une préincubation dans une solution de Glycocollate de Cuivre en tampon acétate à pH5 et une incubation dans la même solution à laquelle a été ajouté l'Iodure d'Acétylthiocholine, pendant trente minutes, puis un virage dans une solution fraîche et diluée (1\%) de sulfure d'ammonium. À l'état normal, chez l'adulte, les appareils sous-neuraux sont formés de quatre à six cupules, ou gouttières, finement striées inscrites dans une zone ovale de trente ou quarante microns dans son plus grand diamètre, pour des fibres musculaires de 40 à 60 microns de diamètre (Figure 1). Il existe en fait une relation linéaire entre la taille et la surface des appareils sous-neuraux et le diamètre des fibres musculaires sous-jacentes.

3. La technique d'imprégnation métallique la plus communément utilisée reste celle de BielchowskyGros, sur matériel fixé par le formaldéhyde et débité 
en coupes de 50 à 100 microns incubées dans une solution à $10 \%$ de Nitrate d'Argent ammoniacal. Cette technique permet de visualiser les neurites terminaux (un par plaque à l'état normal) et l'arborisation nerveuse terminale faite de deux à quatre neurites très fins, terminés parfois par un petit anneau. Une technique d'imprégnation à l'Argent selon Bodian peut être combinée à la mise en évidence de l'activité cholinestérasique des appareils sous-neuraux, selon une méthode initialement proposée par S. Manolov et adaptée par M. Fardeau (Figure 2) [5].

4. Aujourd'hui, cet ensemble de techniques classiques a été remplacé par l'analyse en microscopie confocale après marquage des éléments pré - et post-synaptiques par des anticorps ou toxines spécifiques [6] : $\alpha$-bungarotoxine-tétrarhodamine pour

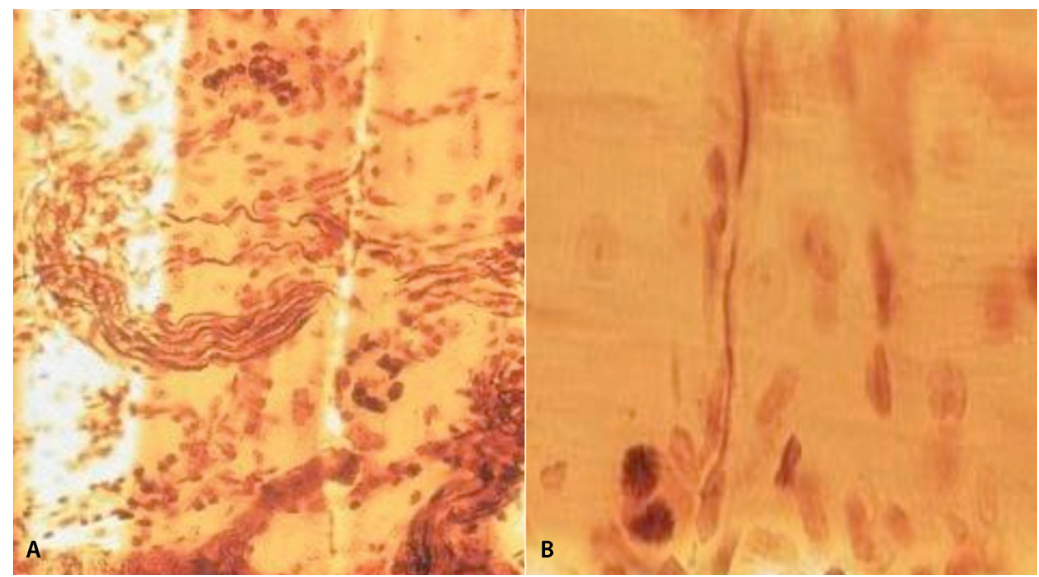

Figure 2

Technique de S. Manolov modifiée [5].

A. Muscle humain normal (muscle deltoïde). B. Plaque motrice de réinnervation (biopsie provenant d'un patient atteint de SLA).
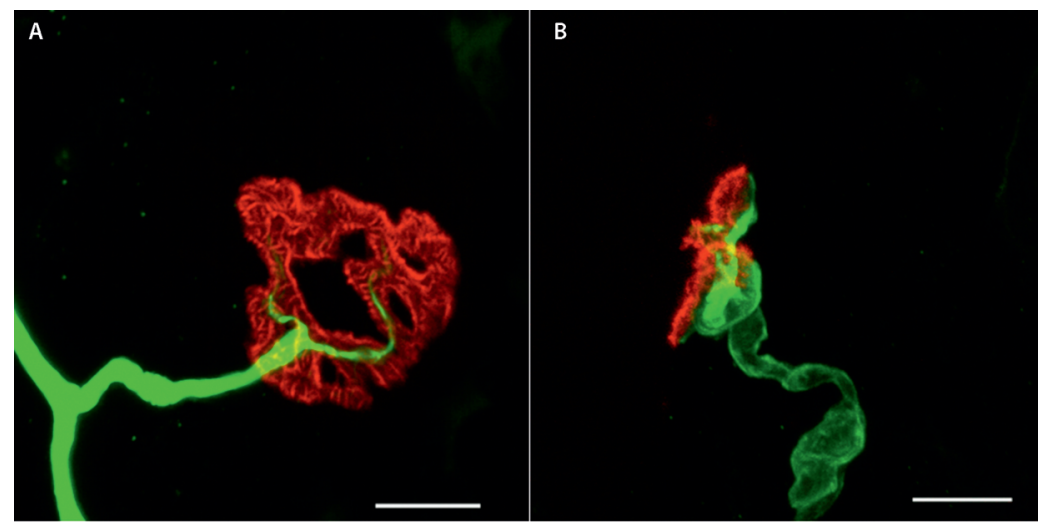

Figure 3

Jonction neuromusuclaire humaine normale. (A) L'axone terminal s'enchasse dans la totalité des gouttières synaptiques (neurofilament en vert et Récepteur Cholinergique en rouge ; image de gauche) et $(\mathrm{B})$ on observe une cellule de Schwann terminale par gouttières synaptique (protéine $\mathrm{S} 100$ en vert et RACh en rouge, image de droite). Échelle : $10 \mu \mathrm{m}$. les sous-unités alpha du récepteur cholinergique; anticorps anti-neurofilaments $(68 \mathrm{kDa}, 165 \mathrm{kDa}$ et $200 \mathrm{kDa}$ ) pour le marquage de l'axone terminal et de ses ramifications; fasciculine couplée à la fluorescéine, pour le marquage de l'acétylcholinestérase dans la fente synaptique. Un anticorps anti-S100 peut être utilisé pour marquer la cellule de Schwann qui coiffe l'arborisation nerveuse terminale. On met ainsi en évidence les différentes composantes de la jonction neuromusculaire (Figure 3).

\section{Résultats en pathologie humaine}

1. Dans les atteintes neuropathiques, la diminution et l'altération des axones terminaux vont de pair avec un processus plus ou moins intense de bourgeonnement ("sprouting") collatéral. Il s'ensuit de grandes variations dans la visualisation des neurites terminaux, ainsi qu'une grande variabilité de taille des appareils sous-neuraux. Ceux-ci sont souvent réduits à une digitation, plus ou moins volumineuse, correspondant à des plaques motrices de réinnervation (Figure 2B). Sur les préparations au Bleu de Méthylène, il est possible d'établir un rapport d'innervation terminal - rapport entre le nombre d'axones terminaux visibles et le nombre de fibres musculaires innervées ; ce rapport est supérieur à 1 dans les atteintes neuropathiques.

2. Dans les atteintes dystrophiques musculaires, les axones terminaux sont aisément visualisés et le rapport d'innervation terminal au voisinage de 1 ; il existe une grande variabilité de taille des appareils sous-neuraux, dont la structure fine est conservée. Dans certaines dystrophies (dystrophie myotonique) il a été noté une modification de la corrélation existant normalement entre les dimensions des appareils sous-neuraux et le diamètre des fibres musculaires sous-jacentes [7].

3. Dans les syndromes myasthéniques congénitaux (SMC), l'analyse de l'IMT et des différents composants des plaques motrices est essentielle [8]. L'un de ces syndromes peut être caractérisé morphologiquement, l'absence d'acétylcholinestérase par déficience de la "queue" collagénique $(\mathrm{Col} \mathrm{Q})$ sur laquelle sont greffées normalement les tétramères d'AChE, alors que les neurites terminaux sont normalement présents ; ceci implique bien évidemment la normalité des contrôles effectués en parallèle (Figure 4). Ces syndromes de déficience en AChE sont aujourd'hui parfaitement caractérisés au niveau génétique moléculaire [9]. D’autres syndromes myasthéniques congénitaux comportent des anomalies du dispositif d'innervation terminale, en particulier les syndromes avec déficience en agrine [10], 


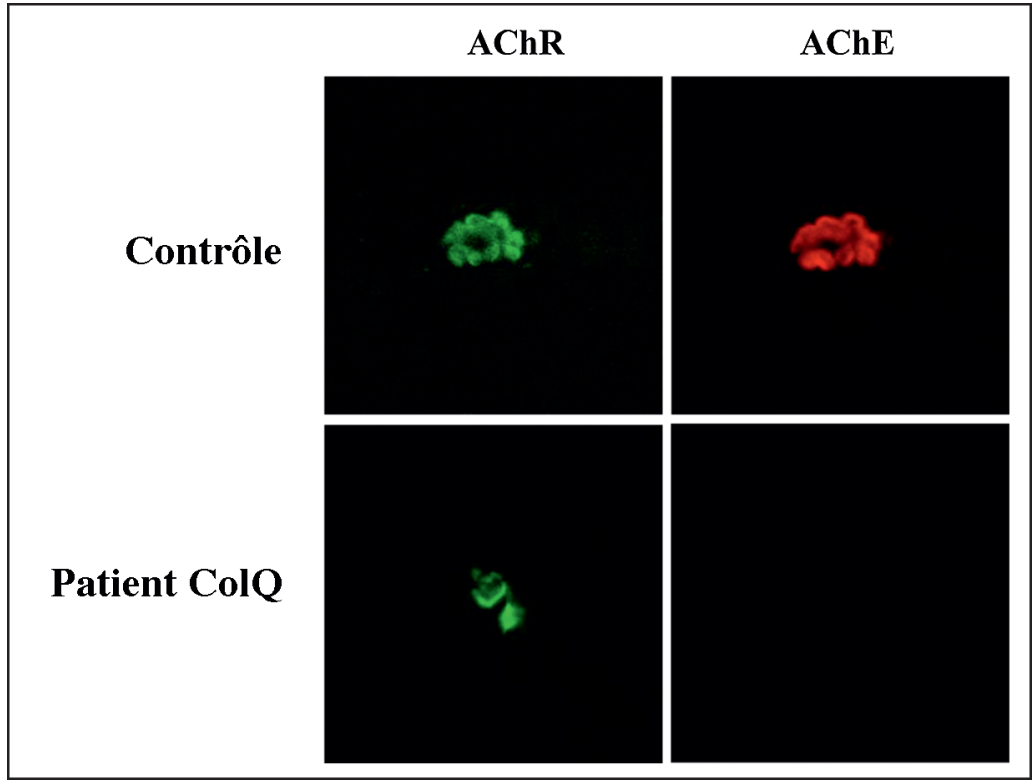

Figure 4

Jonction neuromusuclaire d'un patient avec mutation dans le gène codant ColQ. Présence de co-marquage AChR (vert), AChE (rouge) chez un patient contrôle (images du haut). Chez un patient muté en ColQ, absence de marquage $\mathrm{AChE}$ (rouge) à la jonction neuromusculaire. Échelle : $10 \mu \mathrm{m}$.

molécule sécrétée par les neurites terminaux et essentielle pour la formation ou le remodelage des jonctions neuromusculaires; d'où la présence simultanée dans ce SMC de plaques dénervées et de plaques " remodelées ", avec une diminution du nombre de récepteurs cholinergiques de l'ordre de $50 \%$. Enfin, dans les myasthénies auto-immunes, il a été montré depuis longtemps qu'il existait une simplification des appareils sous-neuraux avec diminution du nombre et de la profondeur des plis sous-neuraux, alors que le dispositif d'innervation terminale n'était, en règle, pas modifié [11].
On the technics of human muscle biopsies (II) Analysis of the terminal motor innervation and endplates in human pathology An historical overview

\section{LIENS D'INTÉRÊT}

Les auteurs déclarent n'avoir aucun lien d'intérêt concernant les données publiées dans cet article.

\section{RÉFÉRENCES}

1. Coërs C, Woolf AL. The innervation of muscle: a biopsy study. Oxford : Blackwell, 1959.

2. Couteaux R, Taxi J. Recherches histochimiques sur la distribution des activités cholinestérasiques au niveau de la synapse myoneurale. Arch Anat Microsc Morphol Exp 1952 ; 41 : 352-92.

3 . Fardeau M. Technics and results of the study of motor endplates in the pathology of skeletal muscle. Rev Neurol (Paris) $1960 ; 103: 30-40$.

4. Engel AG, Lambert EH, Gomez MR. A new myasthenic syndrome with end-plate acetylcholinesterase deficiency, small nerve terminals, and reduced acetylcholine release. Arch Neurol 1977 ; $1: 315-30$.

5. Fardeau M. Simultaneous staining of the terminal motor innervation and the subneural apparatus (S. Manolov's technic). Importance for diagnosis in neuro-muscular pathology. Rev Neurol (Paris) 1964 ; 111 : 501-6.

6. Sanes JR, Lichtman JW. Development of the vertebrate neuromuscular junction. Annu Rev Neurosci 1999; 22 : 389-442.

7. Fardeau M, Tomé FMS. Symposium Inserm $n^{\circ} 13$. In : Taxi J, ed. Paris : Éditions Inserm, $1980: 287-97$.

8. Chevessier F, Faraut B, Ravel-Chapuis A, et al. MUSK, a new target for mutations causing congenital myasthenic syndrome. Hum Mol Genet 2004 ; 13 : 3229-40.

9. Donger C, Krejci E, Serradell AP, et al. Mutation in the human acetylcholinesterase-associated collagen gene, COLQ, is responsible for congenital myasthenic syndrome with end-plate acetylcholinesterase deficiency (Type Ic). Am J Hum Genet 1998; 63: 967-75.

10. Huzé C, Bauché S, Richard P, et al. Identification of an agrin mutation that causes congenital myasthenia and affects synapse function. Am J Hum Genet 2009; 85 : 155-167.

11. Fardeau M, Godet-Guillain J, Chevallay M. Ultrastructural changes of the motor end-plates in myasthenia gravis and myasthenic syndrome. Proceedings of the $\mathrm{X}^{\text {th }}$ International Congress of Neurology. Amsterdam : Excerpta Medica, 1972. 